\section{$M$-Channel Compactly Supported Biorthogonal Cosine-Modulated Wavelet Bases}

\author{
S. C. Chan, Y. Luo, and K. L. Ho
}

\begin{abstract}
In this correspondence, we generalize the theory of compactly supported biorthogonal two-channel wavelet bases to $M$-channel. A sufficient condition for the $M$-channel perfect reconstruction filter banks to construct $M$-channel biorthogonal bases of compactly supported wavelets is derived. It is shown that the construction of biorthogonal $M$-channel wavelet bases is equivalent to the design of a $M$-channel perfect reconstruction filter bank with some added regularity conditions. A family of $M$-channel biorthogonal wavelet bases based on the cosinemodulated filter bank (CMFB) is proposed. It has the advantages of simple design procedure, efficient implementation, and good filter quality. A new method for imposing the regularity on the CMFB's is also introduced, and several design examples are given.
\end{abstract}

\section{INTRODUCTION}

Wavelets are functions generated from the dilations and translations of one basic function called the wavelet function [2], [3], [6]. The Haar system is considered the earliest example of such wavelet bases [2]. Grossmann and Morlet [1] were the first to construct a wavelet function in the square integrable real space $H^{2}$. Recently, orthonormal wavelet bases have been studied extensively both in the mathematical and signal processing communities [2]-[8], [18]. In signal analysis, the wavelet transform [3], which is a representation of a signal in terms of a set of wavelet basis functions, allows the signal to be analyzed in different resolutions or scales. The wavelet transform makes a different tradeoff in the time-frequency plane as compared with the short-time Fourier transform. It has better time resolution in high frequency and better frequency resolution in low frequency. This property is very useful to detect discontinuity in nonstationary signals, which usually have slowly varying components with transient high-frequency spikes.

Multiresolution approaches have been popular in the computer vision community. Mallat defines an important concept of multiresolution analysis and wavelet bases [4]. The theory of wavelets is also closely related to that of multirate perfect reconstruction (PR) filter banks. Daubechies constructed compactly supported dyadic orthonormal wavelets based on iterations of two-channel PR orthogonal filter banks with added regularity condition [2]. Since twochannel orthogonal PR filter banks cannot have nontrivial linear-phase solution, more general biorthogonal filter banks were studied [5], [6]. This approach has further been extended to $M$-channel orthonormal wavelet bases [7], [8]. It is shown that any square integrable signal can be expanded in terms of dilations and translations of $M-1$ functions, $\Psi_{i}^{(j, k)}(x), i=1,2, \cdots, M-1$, which are called the $M$ channel wavelets. For a large class of signals, $M$-channel wavelet decomposition gives a more compact representation than the dyadic one [7]. In addition, the discrete wavelet transform provides a good approximation to the KLT of several processing [17]. Like the dyadic

Manuscript received February 15, 1997; revised November 30, 1997. This work is supported by the Hong Kong Research Grants Council and the CRCG of the University of Hong Kong. The associate editor coordinating the review of this paper and approving it for publication was Ali N. Akansu.

The authors are with the Department of Electrical Engineering, University of Hong Kong, Hong Kong (e-mail: scchan@eee.hku.hk; yluo@eee.hku.hk; klho@eee.hku.hk).

Publisher Item Identifier S 1053-587X(98)02512-4. case, it is possible to obtain $M$-channel wavelet bases from $M$ channel PR orthogonal filter banks with added regularity condition.

In this correspondence, we will derive a sufficient condition for the $M$-channel PR filter banks to construct $M$-channel biorthogonal bases of compactly supported wavelets. It is found that the lowpass filters in the PR filter bank have to satisfy similar regularity condition as in the orthogonal case, and the bandpass and highpass filters have to satisfy the admissibility condition. The design of $M$-channel wavelet bases is considerably more difficult than the two-channel case due to the large number of design parameters in the $M$ channel PR filter bank and the difficulty in meeting the regularity condition exactly. In this correspondence, we propose to use cosinemodulated filter banks (CMFB's) [11]-[13], [16], [19] to construct such a wavelet basis. The advantages of the CMFB are its low design and implementation complexities, good filter quality, and ease in imposing the regularity condition. Nguyen and Koilpillai [13] have also considered the design of orthonormal cosine-modulated wavelets using the orthogonal CMFB. The regularity conditions are imposed as additional constraints in the optimization. Gopinath [14] classified the modulated filter banks according to the type of the discrete cosine or sine transform with which they are associated and generalized the results of modulated wavelet tight frames.

The outline of the paper is as follows. In Section II, we shall briefly review the theory of the $M$-channel PR filter banks. Section III is devoted to an overview of the $M$-channel wavelet bases. In Section IV, the sufficient condition for the $M$-channel PR filter banks to construct $M$-channel biorthogonal bases of compactly supported wavelets is derived. The theory and design of the CMFB is discussed in Section V. A new method for imposing the regularity condition on the CMFB and several design examples are given in Section VI. Finally, we summarize our results in the conclusion.

\section{THEORY OF THE $M$-ChANNEL PR FILTER BANKS}

Fig. 1 shows the structure of an $M$-channel uniform filter bank with $h_{i}(n)$ and $g_{i}(n)$ as the analysis and synthesis filters, respectively. A filter bank is said to be a PR filer bank if the input and output are equal except for a delay, [i.e., $y(n)=x\left(n-n_{d}\right)$ ].

For perfect reconstruction, $h_{i}(n)$ and $g_{i}(n)$ have to satisfy certain PR conditions. These conditions can either be expressed in the time domain or in the $Z$-transform domain. For convenience, we shall give the one in the time domain.

The filter bank has the PR property if it satisfies

$$
\sum_{n}\left[\sum_{i=0}^{M-1} h_{i}\left(M n+n_{1}\right) g_{i}\left(-M n-n_{2}\right)\right]=\delta\left(n_{1}-n_{2}\right) .
$$

Another equivalent condition is given by

$$
\sum_{n} h_{i}(n) g_{j}(-M \ell-n)=\delta(\ell) \delta(i-j) .
$$

For notion convenience, we shall introduce the mirror image $\tilde{g}_{i}(n)$ of $g_{i}(n)$ as

$$
\tilde{g}_{i}(n)=g_{i}(-n) .
$$

Equations (2.1) and (2.2) can then be rewritten as

$$
\sum_{n}\left[\sum_{i=0}^{M-1} h_{i}\left(M n+n_{1}\right) \tilde{g}_{i}\left(M n+n_{2}\right)\right]=\delta\left(n_{1}-n_{2}\right)
$$




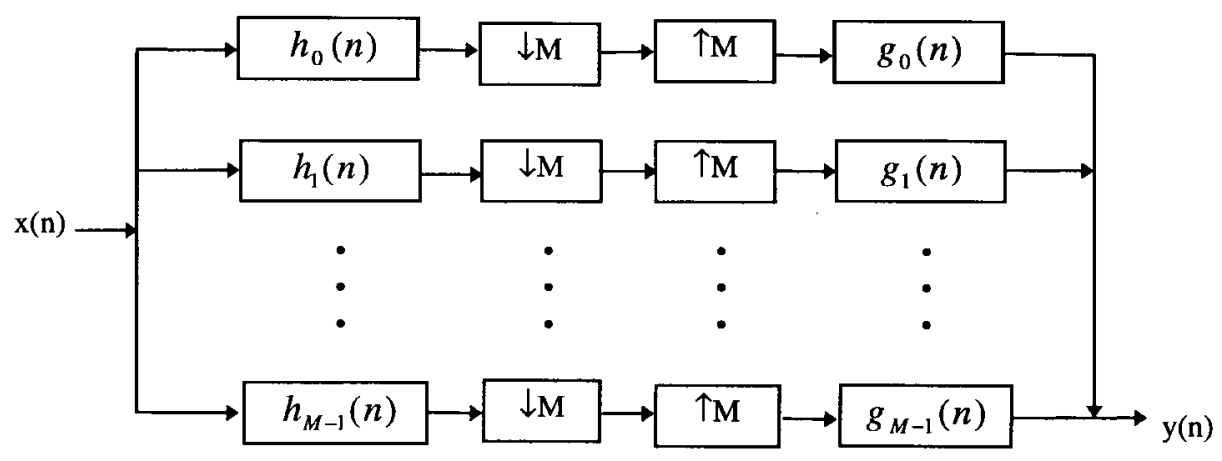

(a)

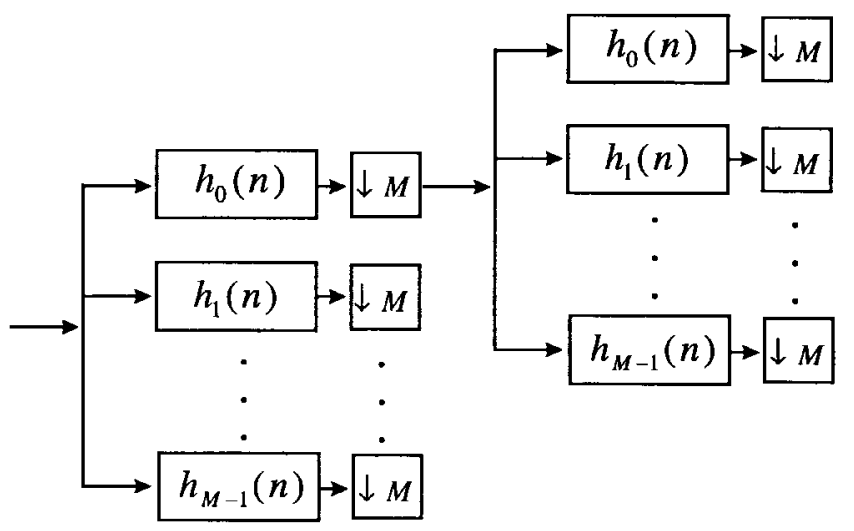

(b)

Fig. 1. (a) $M$-channel uniform filter bank and (b) Two-level $M$-channel tree-structured analysis filter bank.

and

$$
\sum_{n} h_{i}(n) \tilde{g}_{j}(M \ell+n)=\delta(\ell) \delta(i-j)
$$

If the filter bank is orthogonal, $h_{i}(n)$ and $g_{i}(n)$ will be time reverses of each other. The PR condition is further simplified to

$$
\sum_{n}\left[\sum_{i=0}^{M-1} h_{i}\left(M n+n_{1}\right) h_{i}\left(M n+n_{2}\right)\right]=\delta\left(n_{1}-n_{2}\right)
$$

and

$$
\sum_{n} h_{i}(n) h_{j}(M \ell+n)=\delta(\ell) \delta(i-j)
$$

\section{Overview OF $M$-ChanNel WAVELETS}

The theory of wavelets is closely related to that of multirate filter banks [6], [8]. It has been shown that discrete dyadic wavelets can be obtained from two-channel PR filter banks [2], [5], [6] with added regularity condition. Here, we shall consider the general case of $M$ channel biorthogonal wavelet bases. There will be two dual bases, each generated from a set of wavelet functions. First of all, we start with the discrete-time Fourier transforms (scaled by $M^{-1 / 2}$ ) of $h_{0}(n)$ and $\tilde{g}_{0}(n)$

$$
\begin{aligned}
& H_{0}(\omega)=M^{-1 / 2} \sum_{n} h_{0}(n) e^{-j n \omega} \\
& G_{0}(\omega)=M^{-1 / 2} \sum_{n} \tilde{g}_{0}(n) e^{-j n \omega} .
\end{aligned}
$$

By iterating these discrete filters, it is possible to define the Fourier transform $\Phi(\xi)$ and $\tilde{\Phi}(\xi)$ of the scaling function $\phi(x)$ and its dual $\tilde{\phi}(x)$ by using the infinite products

$$
\Phi(\xi)=(2 \pi)^{-1 / 2} \prod_{j=1}^{\infty} H_{0}\left(M^{-j} \xi\right)
$$

and

$$
\tilde{\Phi}(\xi)=(2 \pi)^{-1 / 2} \prod_{j=1}^{\infty} G_{0}\left(M^{-j} \xi\right) .
$$

These infinite products can only converge if

$$
H_{0}(0)=G_{0}(0)=1 .
$$

Equations (3.3) and (3.4) will then converge uniformly and absolutely on compact sets to $\Phi(\xi)$ and $\tilde{\Phi}(\xi)$, which are well-defined $C^{\infty}$ functions. In addition, from the Paley-Wiener theorem, it can be shown that the infinite product is an entire function of exponential type, and it is a Fourier transform of a distribution with support in $\left[N_{1}, N_{2}\right]$ [5]. From (3.3) and (3.4), we also have

$$
\begin{aligned}
& \Phi(\xi)=H_{0}\left(\frac{\xi}{M}\right) \cdot \Phi\left(\frac{\xi}{M}\right) \\
& \tilde{\Phi}(\xi)=G_{0}\left(\frac{\xi}{M}\right) \cdot \tilde{\Phi}\left(\frac{\xi}{M}\right) .
\end{aligned}
$$

Taking the inverse Fourier transform leads to the well-known twoscale difference equations of $\phi(x)$ and its dual $\tilde{\phi}(x)$ as

$$
\begin{aligned}
& \phi(x)=\sqrt{M} \sum_{n} h_{0}(n) \phi(M x-n) \\
& \tilde{\phi}(x)=\sqrt{M} \sum_{n} \tilde{g}_{0}(n) \tilde{\phi}(M x-n) .
\end{aligned}
$$

For orthonormal wavelets, the scaling function $\phi(x)$ will be identical to its dual. Equations (3.8) and (3.9) tell us that $\phi(x)$ and $\tilde{\phi}(x)$ can 
be written as a linear combination of their contracted (by $M$ ) and shifted versions. Therefore, the space spanned by

$$
\phi^{(j, k)}(x)=M^{-j / 2} \phi\left(M^{-j} x-k\right)
$$

and

$$
\tilde{\phi}^{(j, k)}(x)=M^{-j / 2} \tilde{\phi}\left(M^{-j} x-k\right), \quad j, k \in \epsilon Z
$$

at a given resolution $j$ can be viewed as a multiscale approximation of a signal $f(x)$. To show that $\phi(x)$ and $\tilde{\phi}(x)$ can be used to generate a basis, we need $M-1$ wavelet functions and their duals to describe the remaining "details" in the approximation

$$
\begin{array}{r}
\psi_{i}(x)=\sqrt{M} \sum_{n} h_{i}(n) \phi(M x-n) \\
i=1,2, \cdots, M-1
\end{array}
$$

and

$$
\begin{array}{r}
\tilde{\psi}_{i}(x)=\sqrt{M} \sum_{n} \tilde{g}_{i}(n) \tilde{\phi}(M x-n) \\
i=1,2, \cdots, M-1 .
\end{array}
$$

The functions $\psi_{i}(x)$ and $\tilde{\psi}_{i}(x)$ can only be candidates for generating Riesz bases of wavelets if they satisfy the admissibility condition

$$
\int \frac{\left|\Psi_{i}(\xi)\right|^{2}}{|\xi|} d \xi<\infty \text { and } \int \frac{\left|\tilde{\Psi}_{i}(\xi)\right|^{2}}{|\xi|} d \xi<\infty
$$

where $\Psi_{i}(\xi)$ and $\tilde{\Psi}_{i}(\xi)$ denote the Fourier transforms of $\psi_{i}(x)$ and $\tilde{\psi}_{i}(x)$, respectively. For $\psi_{i}(x), \tilde{\psi}_{i}(x) \in L^{1}(\Re), \Psi_{i}(\xi)$, and $\tilde{\Psi}_{i}(\xi)$ are continuous, and (3.13) implies that

and

$$
(2 \pi)^{1 / 2} \Psi_{i}(0)=\int \psi_{i}(x) d x=0
$$

$$
(2 \pi)^{1 / 2} \tilde{\Psi}_{i}(0)=\int \tilde{\psi}_{i}(x) d x=0 .
$$

Taking the Fourier transform of (3.11) and (3.12), we have

$$
\begin{aligned}
& \Psi_{i}(\xi)=\left[\frac{1}{\sqrt{M}} \sum_{n} h_{i}(n) e^{-j(\xi / M) n}\right] \Phi\left(\frac{\xi}{M}\right) \\
& \tilde{\Psi}_{i}(\xi)=\left[\frac{1}{\sqrt{M}} \sum_{n} \tilde{g}_{i}(n) e^{-j(\xi / M) n}\right] \tilde{\Phi}\left(\frac{\xi}{M}\right) .
\end{aligned}
$$

Since $\Phi(0)$ and $\tilde{\Phi}(0)$ cannot be zero [(3.3)-(3.5)], this leads to the necessary conditions on $H_{i}(z)$ and $G_{i}(z)$

$$
\left[\frac{1}{\sqrt{M}} \sum_{n} h_{i}(n)\right]=H_{i}(0)=0
$$

and

$$
\begin{aligned}
{\left[\frac{1}{\sqrt{M}} \sum_{n} \tilde{g}_{i}(n)\right]=G_{i}(0) } & =0 \\
i & =1,2, \cdots, M-1 .
\end{aligned}
$$

To show that the translated and dilated versions of $\psi_{i}(x)$ and $\tilde{\psi}_{i}(x)$ in (3.11) and (3.12) generate a Riesz basis, we need to show the following.

C1) The collection (3.11) and (3.12) constitutes a frame, i.e., $\exists 0<A \leq B<\infty$ such that

$$
A\|f\|^{2} \leq \sum_{i=1}^{m-1} \sum_{j, k}\left|\left\langle f, \psi_{i}^{(j, k)}\right\rangle\right|^{2} \leq B\|f\|^{2}
$$

and

$$
\tilde{A}\|f\|^{2} \leq \sum_{i=1}^{m-1} \sum_{j, k}\left|\left\langle f, \tilde{\psi}_{i}^{(j, k)}\right\rangle\right|^{2} \leq \tilde{B}\|f\|^{2}
$$

where

$$
\tilde{A}=\frac{1}{A}, \tilde{B}=\frac{1}{B} .
$$

C2) They are linearly independent.

For dyadic orthonormal wavelets, the scaling function $\phi(x)$ is identical to its dual. Since $M$ is equal to two, there is only one wavelet function $\psi(x)$. For the biorthogonal case, there are two scaling functions and wavelet functions. The two conditions in $\mathrm{C} 1$ ) and C2) can be satisfied [5] if the associated multirate filter bank $h_{i}(n)$ and $g_{i}(n)$ have the PR property and the scaling functions that satisfy the decay conditions

$$
|\Phi(\xi)| \leq C(1+|\xi|)^{-1 / 2-\varepsilon}
$$

and

$$
\begin{gathered}
|\tilde{\Phi}(\xi)| \leq C(1+|\xi|)^{-1 / 2-\varepsilon}, \text { for some } C \\
\varepsilon>0 .
\end{gathered}
$$

It had also been proved in [5] that if $H_{0}(\omega)$ and $G_{0}(\omega)$ satisfy the "regularity condition"

$$
H_{0}(z)=\left[\frac{1+z^{-1}}{2}\right]^{L} Q(z)
$$

and

$$
G_{0}(z)=\left[\frac{1+z^{-1}}{2}\right]^{L^{\prime}} Q^{\prime}(z)
$$

then the decay condition is satisfied. If $Q(z)\left(Q^{\prime}(z)\right)$ is bounded above by an appropriate constant, then the regularity of the scaling function (and its dual) can be estimated [5]. We shall come to this decay condition for $M$-channel wavelets in Section IV. It is interesting to note that the admissibility condition is automatically satisfied for $M=2$. In this case, we have

$$
G_{0}(z)=H_{1}(-z) \text { and } G_{1}(z)=-H_{0}(-z) .
$$

Therefore, if $H_{0}(z)$ and $G_{0}(z)$ satisfy the regularity condition, i.e., have multiple zeros at $\omega=\pi$, then $H_{1}(z)$ and $G_{1}(z)$ will have the same number of zeros at $\omega=0$.

For the $M$-channel orthonormal wavelets, $\phi(x)$ is identical to its dual, but there are $M-1$ wavelet functions, $\psi_{i}(x), i=$ $1,2, \cdots, M-1$ [7], [8]. The necessary conditions in (3.17) are also satisfied because for $M$-channel orthogonal filter bank, we have

$$
\sum_{i=0}^{M-1}\left|H_{i}(\omega)\right|^{2}=1 \text {. }
$$

Since $H_{0}(0)=1$, we must have $H_{i}(0)=0, i=1,2, \cdots, M-1$. It had been shown in [8] that if $H_{0}(\omega)$ satisfies the " $K$-regularity condition"

$$
H_{0}(z)=\left[\frac{1+z^{-1}+\cdots+z^{-(M-1)}}{M}\right]^{K} Q(z)
$$

then the decay condition will also be satisfied.

\section{THEORY OF $M$-ChANNEL BIORTHOGONAL WAVELETS}

In this section, we generalize the idea in [5] to the $M$-channel biorthogonal bases of compactly supported wavelets. We shall prove that if the scaling functions of a biorthogonal PR filter bank satisfy the decay conditions (3.19), then the collection (3.11) and (3.12) generates a Riesz basis. In particular, we need to establish (3.18), i.e., they generate a frame, and show that the collection (3.11) and (3.12) is linearly independent. 


\section{A. The Collection Generates a Frame}

Suppose that $\phi(x)$ and $\tilde{\phi}(x)$ satisfy the decay condition (3.19); it can be shown that

A1)

and

$$
\sum_{j, k}\left|\left\langle f_{1}, \psi_{i}^{(j, k)}\right\rangle\right|^{2} \leq C\left\|f_{1}\right\|^{2}
$$

$$
\sum_{j, k}\left|\left\langle f_{2}, \tilde{\psi}_{i}^{(j, k)}\right\rangle\right|^{2} \leq C^{\prime}\left\|f_{2}\right\|^{2}
$$

A2)

$$
\begin{aligned}
f(x) & =\sum_{j, k} \sum_{i=1}^{M-1}\left\langle f, \tilde{\psi}_{i}^{(j, k)}\right\rangle \psi_{i}^{(j, k)} \\
& =\sum_{j, k} \sum_{i=1}^{M-1}\left\langle f, \psi_{i}^{(j, k)}\right\rangle \tilde{\psi}_{i}^{(j, k)} .
\end{aligned}
$$

Due to space limitations, the proof is omitted. Interested readers are referred to [15]. The proof is similar to the two-channel filter bank situation [5].

The upper bound in (3.18) is immediate from (4.1). For the lower bound, we have

$$
\begin{aligned}
\|f\|= & \sup _{\|g\|=1}|\langle f, g\rangle| \\
\leq & \sup _{\|g\|=1} \sum_{j, k} \sum_{i=1}^{M-1}\left|\left\langle f, \psi_{i}^{(j, k)}\right\rangle\right|\left|\left\langle\tilde{\psi}_{i}^{(j, k)}, g\right\rangle\right| \\
\leq & {\left[\sum_{j, k} \sum_{i=1}^{M-1}\left|\left\langle f, \psi_{i}^{(j, k)}\right\rangle\right|^{2}\right]^{1 / 2} } \\
& \cdot \sup _{\|g\|=1}\left[\sum_{j, k} \sum_{i=1}^{M-1}\left|\left\langle\tilde{\psi}_{i}^{(j, k)}, g\right\rangle\right|^{2}\right]^{1 / 2}
\end{aligned}
$$

(By Schwartz the inequality)$$
\leq C\left[\sum_{j, k} \sum_{i=1}^{M-1}\left|\left\langle f, \psi_{i}^{(j, k)}\right\rangle\right|^{2}\right]^{1 / 2} \text {. }
$$

\section{B. The Collection Is Linearly Independent}

We first show that

B1) $\psi_{i}^{(j, k)}$ and $\tilde{\psi}_{i}^{(j, k)}, j, k \in Z$ are linearly independent if and only if

$$
\left\langle\psi_{i}^{(j, k)}, \tilde{\psi}_{i^{\prime}}^{\left(j^{\prime}, k^{\prime}\right)}\right\rangle=\delta\left(j-j^{\prime}\right) \delta\left(k-k^{\prime}\right) \delta\left(i-i^{\prime}\right) .
$$

This is the biorthogonal relation for Riesz bases.

B2) A sufficient condition for (4.3) to hold is

$$
\left\langle\phi^{(0, \ell)}, \tilde{\phi}^{(0, k)}\right\rangle=\delta(k-\ell) .
$$

That is, $\phi(x-\ell)$ and $\tilde{\phi}(x-k)$ are biorthogonal.

In Appendix A, we shall show that (4.4) is satisfied if $\phi(x)$ and $\tilde{\phi}(x)$ satisfy the decay condition (3.19).

\section{Proof:}

B1) If (4.3) is satisfied, then any $f$ in the closed linear span of the $\psi_{i}^{(j, k)}$ with $(i, j, k) \neq\left(i^{\prime}, j^{\prime}, k^{\prime}\right)$ satisfies $\left\langle f, \tilde{\psi}_{i^{\prime}}^{\left(j^{\prime}, k^{\prime}\right)}\right\rangle=$ 0 . It follows that $\psi_{i^{\prime}}^{\left(j^{\prime}, k^{\prime}\right)}$ is not in this closed linear span. From (4.2), we have

$$
\psi_{i^{\prime}}^{\left(j^{\prime}, k^{\prime}\right)}=\sum_{j, k} \sum_{i=1}^{M-1}\left\langle\psi_{i^{\prime}}^{\left(j^{\prime}, k^{\prime}\right)}, \tilde{\psi}_{i}^{(j, k)}\right\rangle \psi_{i}^{(j, k)}
$$

and hence, $\left[1-\left\langle\psi_{i^{\prime}}^{\left(j^{\prime}, k^{\prime}\right)}, \tilde{\psi}_{i^{\prime}}^{\left(j^{\prime}, k^{\prime}\right)}\right\rangle\right] \psi_{i^{\prime}}^{\left(j^{\prime}, k^{\prime}\right)}=$ $\sum_{\substack{j, k \\ i, j, k \neq i, j^{\prime}, k^{\prime}}} \sum_{i=1}^{M-1}\left\langle\psi_{i^{\prime}}^{\left(j^{\prime}, k^{\prime}\right)}, \tilde{\psi}_{i}^{(j, k)}\right\rangle \psi_{i}^{(j, k)}$. If the $\psi_{i}^{(j, k)}$ are linearly independent, then this implies

$$
\left\langle\psi_{i}^{(j, k)}, \psi_{i^{\prime}}^{\left(j^{\prime}, k^{\prime}\right)}\right\rangle=\delta\left(j-j^{\prime}\right) \delta\left(k-k^{\prime}\right) \delta\left(i-i^{\prime}\right) .
$$

B2) First, we shall show that

$$
\left\langle\phi^{(0, k)}, \tilde{\phi}^{(0, \ell)}\right\rangle=\delta_{k \ell} \text { iff }\left\langle\phi^{(j, k)}, \tilde{\phi}^{(j, \ell)}\right\rangle=\delta_{k \ell} .
$$

Equation (4.5) follows from the definition of $\left\langle\phi^{(0, k)}, \tilde{\phi}^{(0, \ell)}\right\rangle$

$$
\left\langle\phi^{(0, k)}, \tilde{\phi}^{(0, \ell)}\right\rangle=\int \phi(x-k) \tilde{\phi}(x-\ell) d x
$$

and the changing of variable $x=M^{-j} u$. Using the relationship between $\phi(x),[\tilde{\phi}(x)]$, and $\psi(x),[\tilde{\psi}(x)]$, we have

$$
\begin{aligned}
\phi^{(j, k)}(x) & =\sum_{n} h_{0}(n) \phi^{(j-1, M k+n)}(x) \\
\psi_{i}^{(j, k)}(x) & =\sum_{n} h_{i}(n) \phi^{(j-1, M k+n)}(x) .
\end{aligned}
$$

We shall verify that (4.3) holds for $j=j^{\prime}$. From (4.6) and (4.7), we have

$$
\begin{aligned}
\left\langle\psi_{i}^{(j, k)}, \tilde{\psi}_{i^{\prime}}^{(j, \ell)}\right\rangle & \\
= & \sum_{n, m} h_{i}(n) \tilde{g}_{i^{\prime}}(m)\left\langle\phi^{(j-1, M k+n)}, \tilde{\phi}^{(j-1, M \ell+m)}\right\rangle \\
= & \sum_{n} h_{i}(n) \tilde{g}_{i^{\prime}}[M(k-\ell)+n]=\delta(k-\ell) \delta\left(i-i^{\prime}\right) \\
& \quad[\text { from (2.5) and (4.5)]. }
\end{aligned}
$$

Similarly, from (4.6) and (4.7)

$$
\begin{aligned}
\left\langle\psi_{i}^{(j, k)}, \tilde{\psi}^{(j, \ell)}\right\rangle & \\
= & \sum_{n, m} h_{i}(n) g_{0}(m)\left\langle\phi^{(j-1, M k+n)}, \tilde{\phi}^{(j-1, M \ell+m)}\right\rangle \\
= & \sum_{n} h_{i}(n) \tilde{g}_{0}[M(k-\ell)+n]=\delta(k-\ell) \delta(i)=0 . \\
& \quad[\text { from (2.5) and (4.5)]. }
\end{aligned}
$$

Since, for $j<j^{\prime}, \tilde{\psi}_{i}^{\left(j^{\prime}, k^{\prime}\right)}$ can be written as a linear combination of $\tilde{\phi}^{(j, \ell)}$, it follows that

$$
\left\langle\psi_{i}^{(j, k)}, \tilde{\psi}_{i}^{\left(j^{\prime}, k^{\prime}\right)}\right\rangle=0, \quad \text { if } j<j^{\prime}
$$

Similarly, since for $j>j^{\prime}, \psi_{i}^{(j, k)}$ can be written as a linear combination of the $\phi^{(j, \ell)}$, it follows that

$$
\left\langle\psi_{i}^{(j, k)}, \tilde{\psi}_{i}^{\left(j^{\prime}, k^{\prime}\right)}\right\rangle=0, \quad \text { if } j>j^{\prime}
$$

This proves B2).

\section{K-Regularity}

In this section, we shall show that if the lowpass analysis and synthesis filters satisfy some regularity condition ( $K$-regularity [7], $[8])$, then the scaling functions and wavelets will satisfy the decay conditions in (3.19).

$H_{0}(\omega)$ is said to be $K$-regular if it can be factored as

$$
H_{0}(z)=\left[\frac{1+z^{-1}+z^{-2}+\cdots+z^{-(M-1)}}{M}\right]^{K} Q(z)
$$




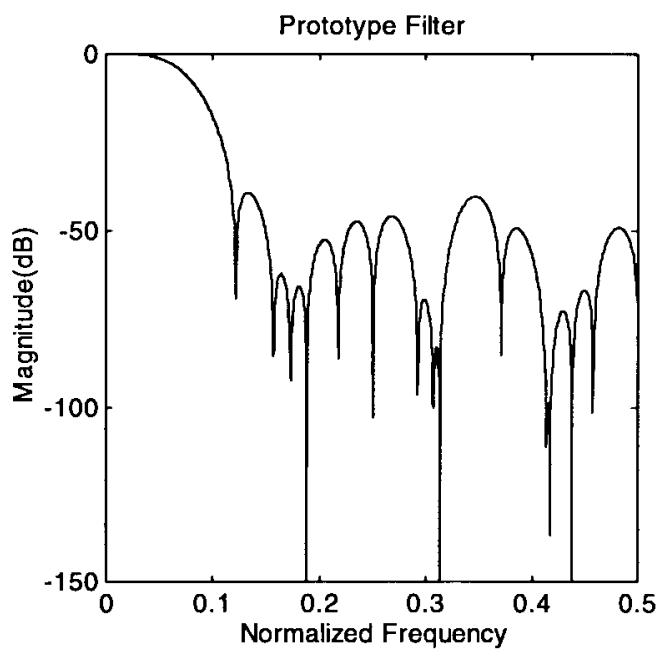

(a)

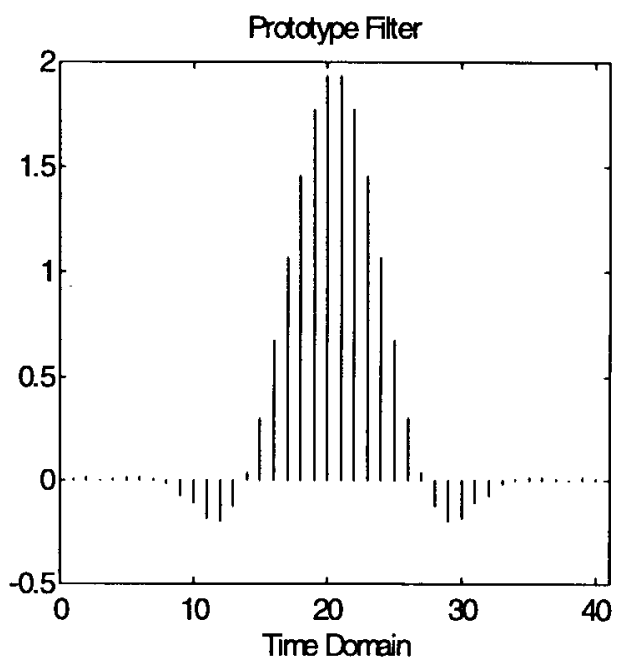

(b)

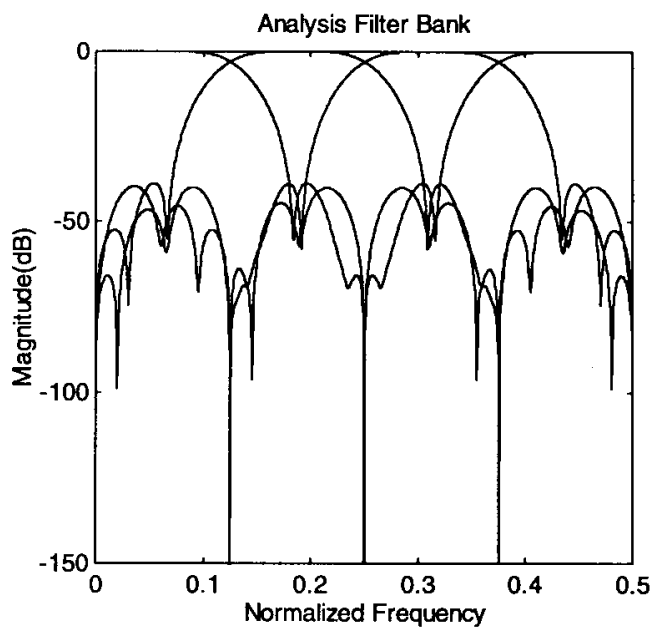

(c)

Fig. 2. Four-channel orthogonal CMFB wavelet bases. Filter length is 40. (a) Prototype filter in frequency domain. (b) In time domain. (c) Analysis filter bank.

where $z=e^{j \omega}$, and $Q(z)$ is a trigonometric polynomial. The precise relationship between $K$-regularity of the scaling filters $\left[h_{0}(n)\right.$ and $\left.g_{0}(n)\right]$ and the smoothness of the scaling functions and wavelets is unknown even in the two-channel case. Using the techniques in [5], it is possible to estimate the regularity of the scaling function. In fact, we can prove the following [15].

If for some value $k \geq 0$

$$
B_{k}=\max _{\xi}\left|Q(\xi) Q(M \xi) \cdots Q\left(M^{k-1} \xi\right)\right|^{1 / k}<M^{L-1 / 2}
$$

then $\Phi(\xi)$ satisfies the decay condition

$$
|\tilde{\Phi}(\xi)| \leq C(1+|\xi|)^{-(1 / 2)-\varepsilon} \text {, with } \varepsilon=L-\frac{1}{2}-\frac{\log B_{k}}{\log M}>0 .
$$

The proof will be given in Appendix B.

In summary, to construct $M$-channel biorthogonal bases of compactly supported wavelets, the lowpass filters of the associated $M$ channel PR biorthogonal filter banks should satisfy the $K$-regularity condition, and the highpass and bandpass filters should satisfy the admissibility condition.

\section{Theory of the Cosine-Modulated Filter BanK}

In this section, we shall introduce the theory of the CMFB and its design procedure. The design procedure will be used in Section VI to construct $M$-channel wavelet bases. More details of CMFB theory can be found in [11]-[13], [16], and [19]. In the CMFB, the analysis filter bank $f_{k}(n)$ and synthesis filter bank $g_{k}(n)$ are obtained by modulating the prototype filters $h(n)$

$$
\begin{array}{r}
f_{k}(n)=h(n) c_{k, n} ; g_{k}(n)=h(n) \bar{c}_{k, n} \\
k=0,1, \cdots, M-1 \\
n=0,1, \cdots, N-1
\end{array}
$$

where $M$ is the number of channels, and $N$ is the length of the filters. The cosine modulation that we use is

$$
c_{k, n}=2 \cos \left[(2 k+1) \frac{\pi}{2 M}\left(n-\frac{N-1}{2}\right)+(-1)^{k} \frac{\pi}{4}\right] .
$$

This modulation is also closely related to the extended lapped transform (ELT) proposed in [12]. It is noted that if $h(n)$ is a linearphase filter, namely, $h(n)=h(N-1-n)$, then $f_{k}(n)$ and $g_{k}(n)$ will be time reverses of each other, and we obtain the orthogonal CMFB. On the other hand, if $h(n)$ is not a linear-phase filter, then we obtain a biorthogonal CMFB. 


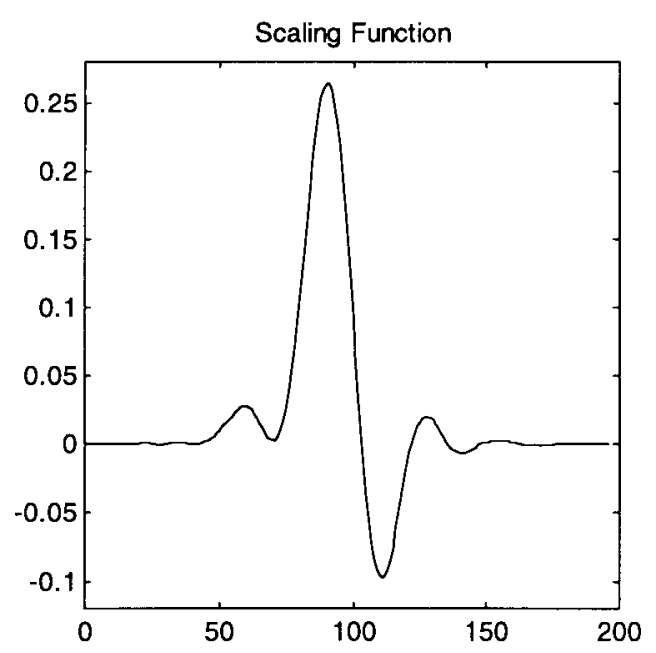

(a)

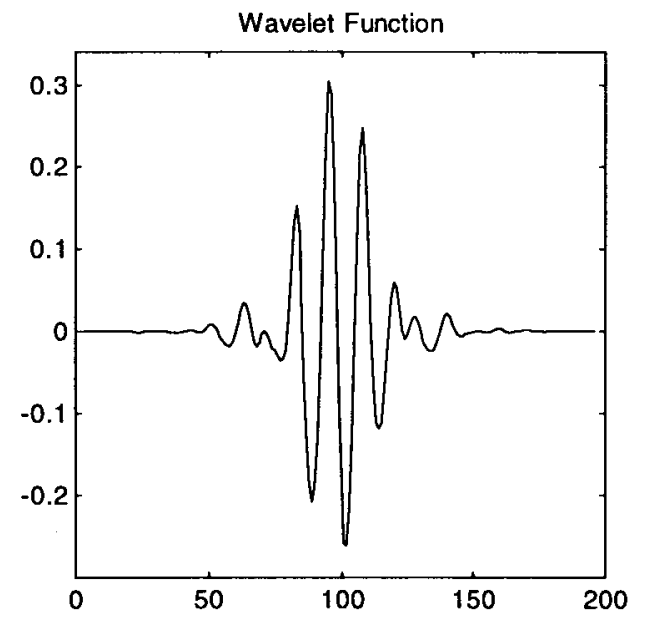

(c)

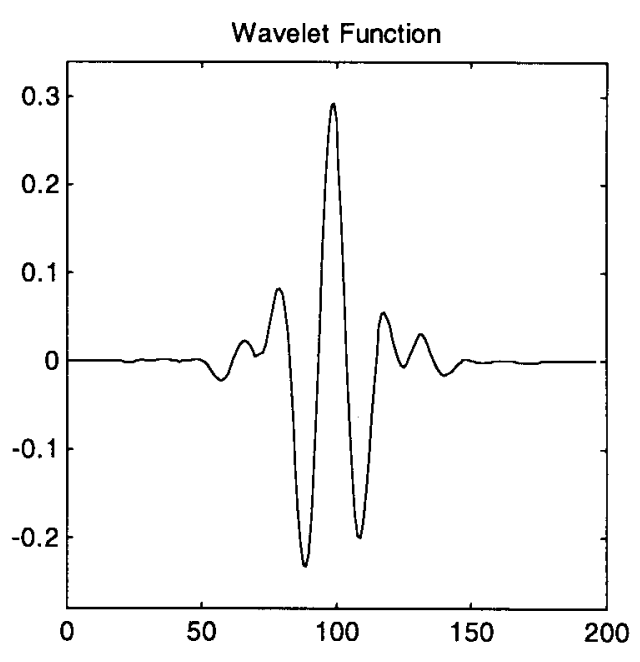

(b)

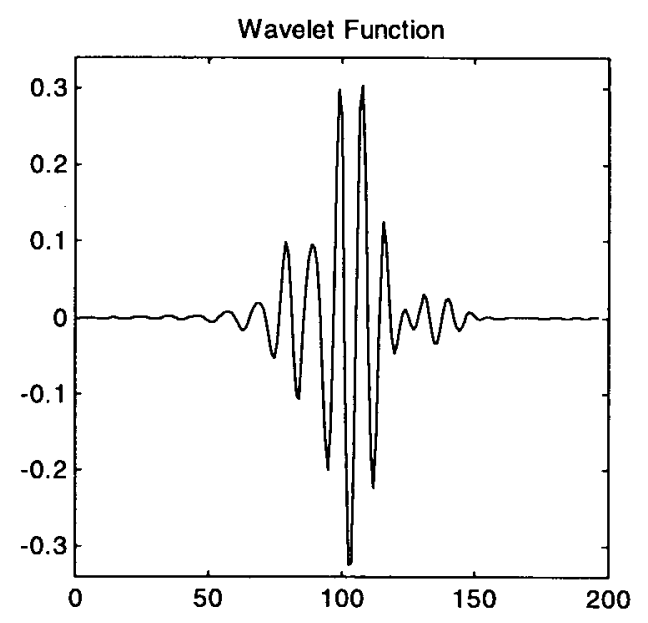

(d)

Fig. 3. Scaling and wavelet functions of four-channel orthogonal CMFB wavelet bases. (a) Scaling function. (b) Wavelet function-1. (c) Wavelet function-2. (d) Wavelet function-3.

Letting $H(z)=\sum_{k=0}^{2 M-1} z^{-k} G_{k}\left(z^{2 M}\right)$ be the type-I polyphase decomposition [9] of the prototype filter, it can be shown [16], [19] that the PR conditions are given by

$$
G_{k}(z) G_{2 M-k-1}(z)+G_{M+k}(z) G_{M-k-1}(z)=\beta z^{-\alpha} .
$$

For orthogonal CMFB, the PR conditions are further simplified to

$$
\tilde{G}_{k}(z) G_{k}(z)+\tilde{G}_{M+k}(z) G_{M+k}(z)=1
$$

where $\tilde{G}_{k}(z)=z^{m-1} G_{2 M-1-k}(z)$. It can be seen that the PR conditions in (5.3) and (5.4) depend only on the prototype filter $h(n)$. There will be $M / 2$ PR conditions when $M$ is even and $\lfloor M / 2\rfloor$ when $M$ is odd. Therefore, the design and implementation complexities can be greatly reduced. In the orthogonal case, the number of free parameters is further reduced by half due to the linear-phase property of the prototype filter.

Since the filter banks are frequency-shifted versions of the prototype filter, the objective function in the optimization is

$$
\Phi=\int_{\omega_{s}}^{\pi}\left|H\left(e^{i \omega}\right)\right|^{2} d \omega
$$

where $\omega_{s}$ is the stopband cutoff frequency whose value should be chosen between $\pi / 2 M$ and $\pi / M$. It is also possible to replace the integral in (5.5) by a summation. It has the advantage of being able to put different weightings to different parts of the stopband and provides more control over the stopband attenuation.

The design problem is formulated as the constrained optimization

$$
\min _{h} \int_{\omega_{s}}^{\pi}\left|H\left(e^{j \omega}\right)\right|^{2} d \omega
$$

subjected to the PR conditions in (5.3) or (5.4) for orthogonal and biorthogonal CMFB.

The design procedure is similar to the one that we had introduced in [16] and the optimization is performed using the NCONF (DNCONF) subroutine of the IMSL library. In the case of biorthogonal CMFB, the linear-phase requirement of the prototype filter is relaxed. Therefore, we have more freedom in choosing its coefficients. In particular, biorthogonal CMFB can be used to realize filter banks with different delays.

\section{The Design of $M$-Channel CMFB Wavelet Bases}

In this section, we are going to construct a family of $M$-channel wavelet bases called cosine-modulated wavelet bases using the CMFB. According to the conditions derived in Section IV, the lowpass analysis (synthesis) filter should satisfy the $K$-regularity condition

$$
F_{0}(z)=C\left[1+z-1+\cdots+z^{-(m-1)}\right]^{K} B(z)
$$




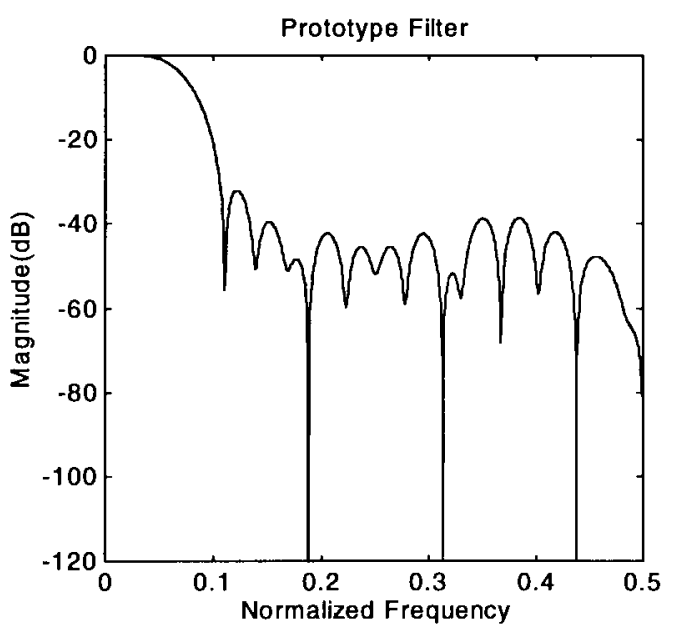

(a)

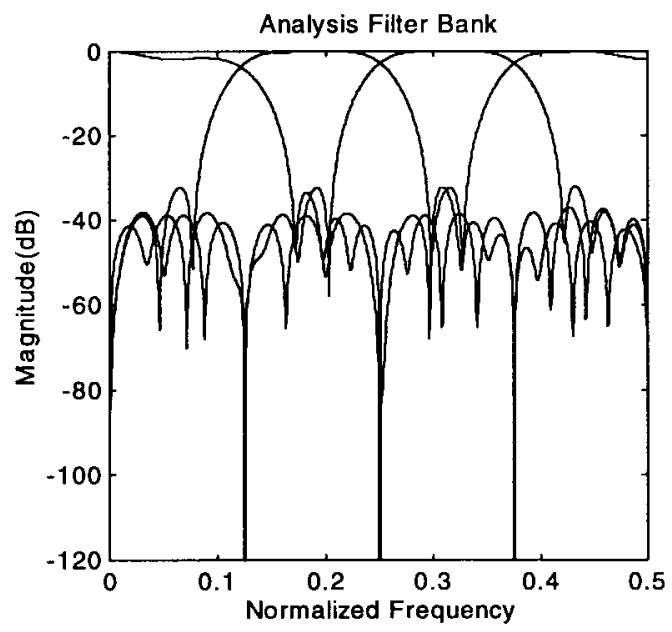

(c)

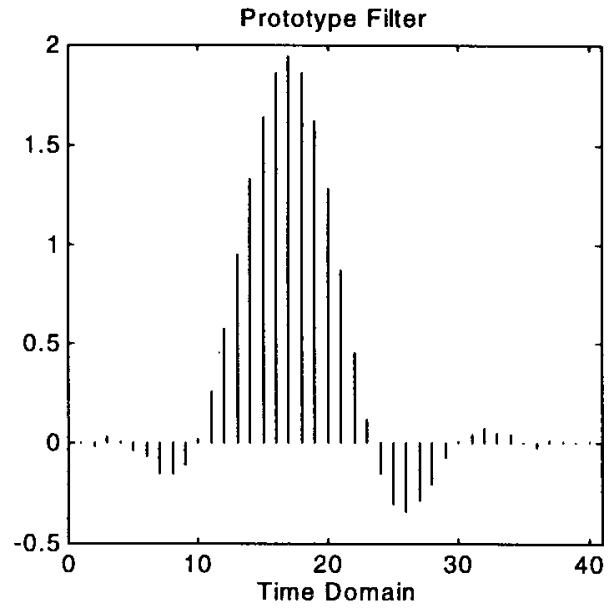

(b)

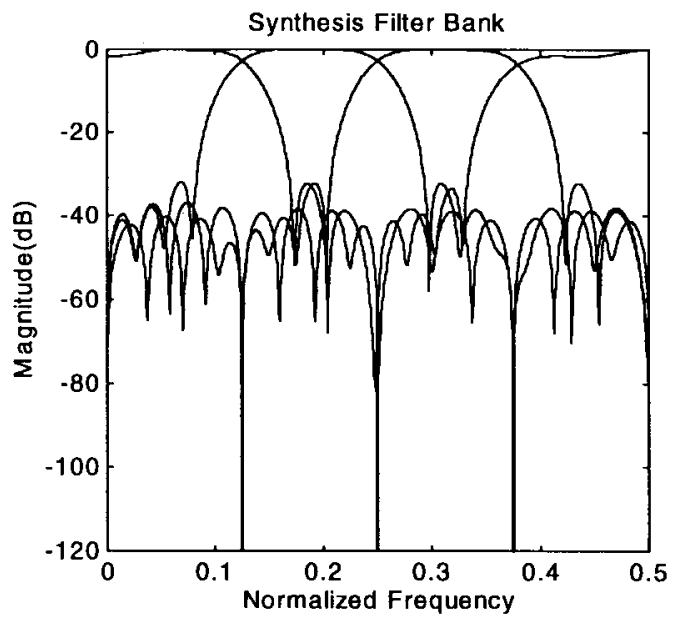

(d)

Fig. 4. Four-channel biorthogonal CMFB wavelet bases. Filter length is 40. (a) Prototype filter in frequency domain. (b) In time domain. (c) Analysis filter bank. (d) Synthesis filter bank.

where $B(z)$ is a polynomial in $z$, and $C$ is a constant. Moreover, all the highpass or bandpass filters should have at least one zero at $\omega=0$. Due to the low design and implementation complexities of the $\mathrm{CMFB}$, it has been used to design compactly supported orthonormal cosine-modulated wavelet bases [13]. Our approach is to decompose the prototype filter $H(z)$ into two parts:

$$
H(z)=Q(z) P(z)
$$

and determine the polynomial $P(z)$ such that after modulation $F_{0}(z)$ will have the required zeros at $\omega_{\ell}=(2 \pi \ell) / M, \ell=1,2, \cdots, M-1$. From (5.1), we note that $f_{0}(n)$ is derived from $h(n)$ using the cosine modulation

$$
\begin{aligned}
f_{0}(n) & =h(n) c_{0, n}=2 h(n) \cos \left[\frac{\pi}{2 M}\left(n-\frac{N-1}{2}\right)+\frac{\pi}{4}\right] \\
& =2 h(n) \cos \left(\frac{\pi}{2 M} n+\varphi\right)
\end{aligned}
$$

where

$$
\varphi=\frac{(M-N+1) \pi}{4 M}, \quad n=0,1, \cdots, N-1 .
$$

Therefore, the frequency response of $F_{0}\left(e^{j \omega}\right)$, is given by

$$
F_{0}\left(e^{j \omega}\right)=e^{j \varphi} H\left[e^{j(\omega-\pi / 2 M)}\right]+e^{-j \varphi} H\left[e^{j(\omega+\pi / 2 M)}\right] .
$$

It means that $H\left(e^{j \omega}\right)$ is shifted along the frequency axis by $\pi / 2 M$ and $-\left(\pi / 2 M\right.$. If $\omega_{\ell}$ are zeros of $F_{0}\left(e^{j \omega}\right)$, then the right-hand side of (6.4) should also be zero. This will be the case if $H\left(e^{j \omega}\right)$ have zeros at $\omega_{\ell} \pm(\pi / 2 M)$. Therefore, for the $M$-channel CMFB to have the $K$-vanishing moment, $H\left(e^{j \omega}\right)$ should have zeros of order $K$ at $\omega_{m}=(4 m \pm 1) \pi / 2 M, m=1,2, \cdots, M-1$.

Hence the polynomial $P(z)$ is given by

$$
\begin{aligned}
P(z)= & \left\{\prod_{m=1}^{M-1}\left[z^{-1}-e^{j(4 m+1 / 2 M) \pi}\right]\right. \\
& \left.\cdot\left[z^{-1}-e^{j(4(M-m)-1 / 2 M) \pi}\right]\right\}^{K} .
\end{aligned}
$$

By multiplying $P(z)$ with $Q(z)$ that contains the free parameters, the prototype filter $H(z)$ will always satisfy the regularity condition of a $M$-channel wavelet.

This design procedure can be used in both orthogonal and biorthogonal cases because the regularity condition is identical in both cases. It is interesting to note that due to the frequency shift property of CMFB, all the highpass and bandpass filters will have the same number of zeros at $\omega=0$ and satisfy (3.17) automatically. A number of orthogonal and biorthogonal cosine-modulated wavelet bases with different values of $M$ and $K$ were designed. Fig. 2(a)-(c) shows an example of the prototype filter and analysis filter bank of an orthogonal wavelet bases. The length of the filters is $N=40$ with 


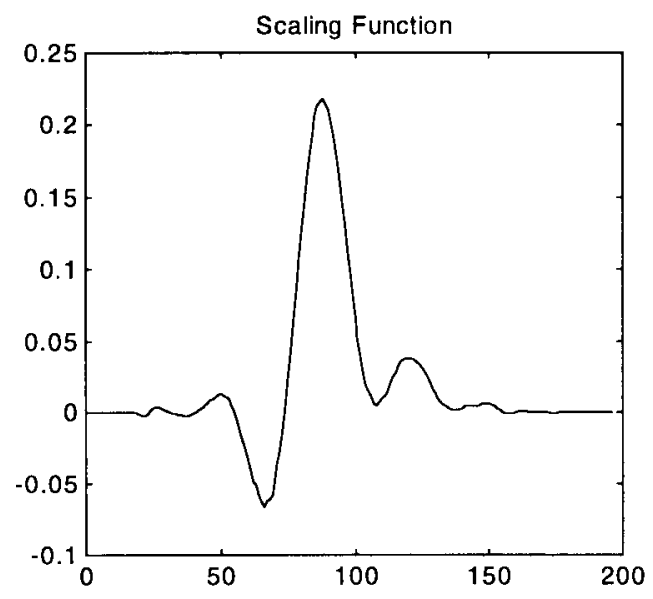

(a)

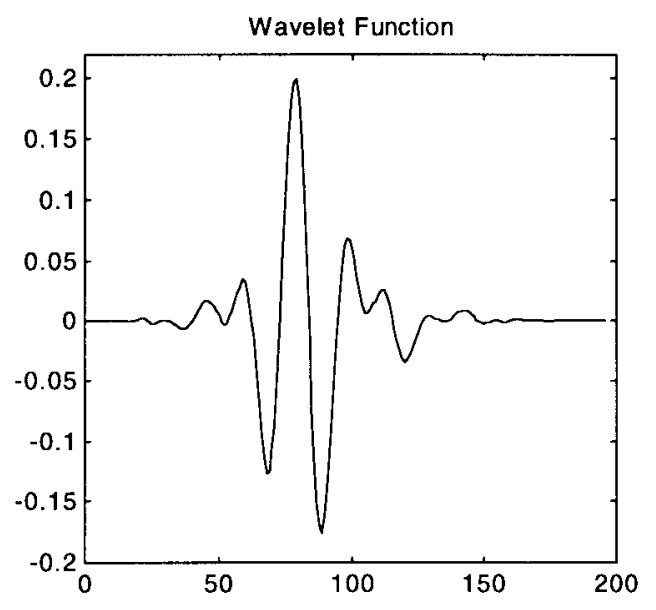

(c)

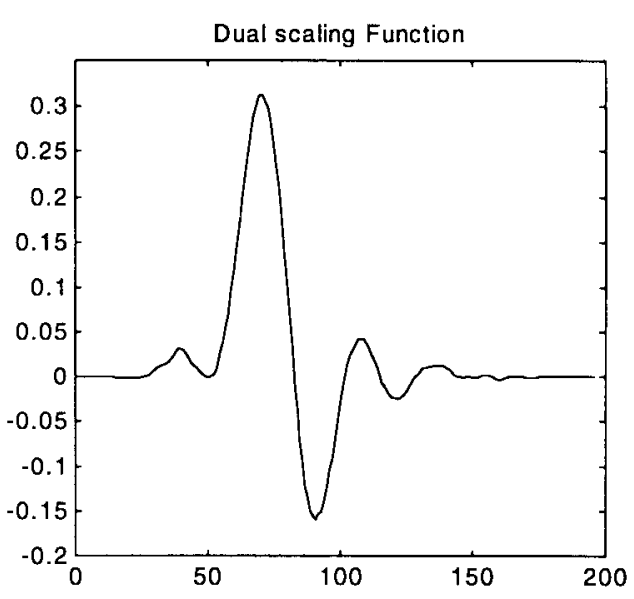

(b)

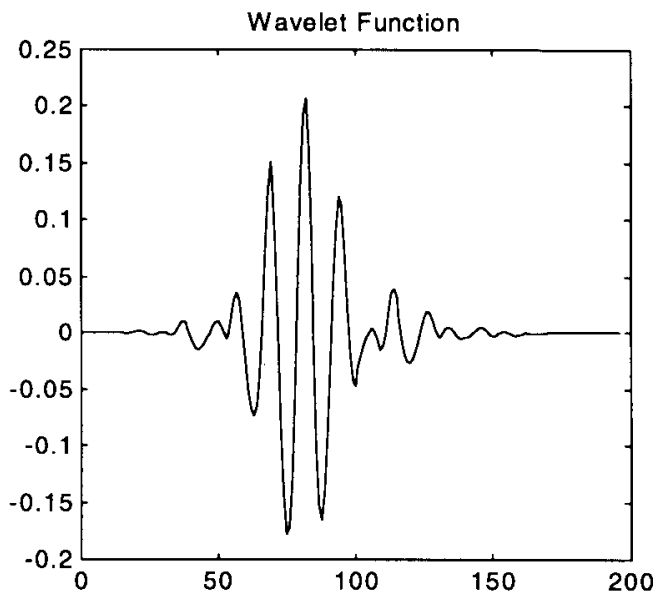

(d)

Fig. 5. Scaling and wavelet functions of four-channel biorthogonal CMFB wavelet bases. (a) Scaling function. (b) Dual scaling function. (c) Wavelet function-1. (d) Wavelet function-2.

$M=4$ and $K=1$. Fig. 3 shows the corresponding scaling and wavelet functions. Figs. 4 and 5 show another example of a fourchannel biorthogonal CMFB and its corresponding wavelet bases. It can be seen from Figs. 2(a) and 4(a) that the prototype filters have the desired zeros at $3 \pi / 8,5 \pi / 8,7 \pi / 8$ and their conjugates. Due to the regularity condition, the degree of freedom in $Q(z)$ is reduced (for the same filter length), and the cutoff frequency of the prototype filter is enlarged. As a result, the overlap between adjacent analysis/synthesis filters is also increased. The scaling and wavelet functions in Figs. 3 and 5 are obtained from iterating the corresponding two-level tree-structured filter bank [Fig. 1(b)].

It should be noted that $K$-regularity is only a sufficient condition to satisfy the decay condition in (3.19). Therefore, wavelets with higher $K$-regularity are not necessarily smoother than other wavelets with lower $K$-regularity if the filter lengths of both systems are the same. Hence, there is a tradeoff between the number of free parameters in the polynomial $Q(z)$ and the order of zeros that are imposed.

\section{CONCLUSION}

In this correspondence, a theory of $M$-channel biorthogonal bases of compactly supported wavelets constructed from $M$-channel PR filter banks is presented. It is found that the lowpass filters in the PR filter bank have to satisfy a similar regularity condition as in the orthogonal case, and the bandpass and highpass filters have to satisfy the admissibility condition. The cosine-modulated filter bank
(CMFB) is used to construct a family of such wavelet bases that has the advantages of low design and implementation complexities, good filter quality, and ease in imposing the regularity and admissibility condition. A new method for imposing the regularity on the CMFB is also introduced, and several design examples are given.

\section{APPENDIX A}

Here, we shall show that if both $H_{0}(\xi)$ and $G_{0}(\xi)$ satisfy the condition (3.19), then $\phi(x-\ell)$ and $\tilde{\phi}(x-\ell)$ are biorthogonal. The proof is similar to the two-channel case in [5].

Proof: First, we define the sequence

$$
\begin{aligned}
& U_{n}(\xi)=\frac{1}{\sqrt{2 \pi}}\left[\prod_{j=1}^{n} H_{0}\left(M^{-j} \xi\right)\right] \chi_{[-\pi, \pi]}\left(M^{-n} \xi\right) \\
& \tilde{U}_{n}(\xi)=\frac{1}{\sqrt{2 \pi}}\left[\prod_{j=1}^{n} \tilde{H}_{0}\left(M^{-j} \xi\right)\right] \chi_{[-\pi, \pi]}\left(M^{-n} \xi\right) .
\end{aligned}
$$

It can be proved that

$$
\int u_{n}(x) \tilde{u}_{n}(x-\ell) d x=\delta_{\ell 0}
$$




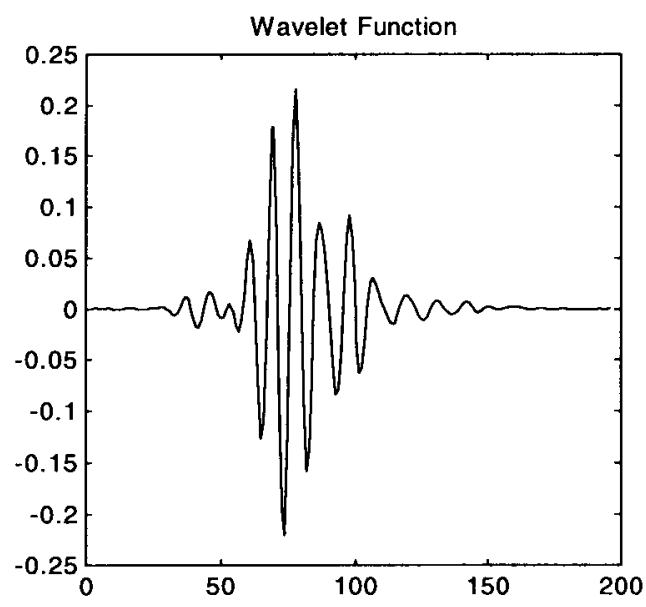

(e)

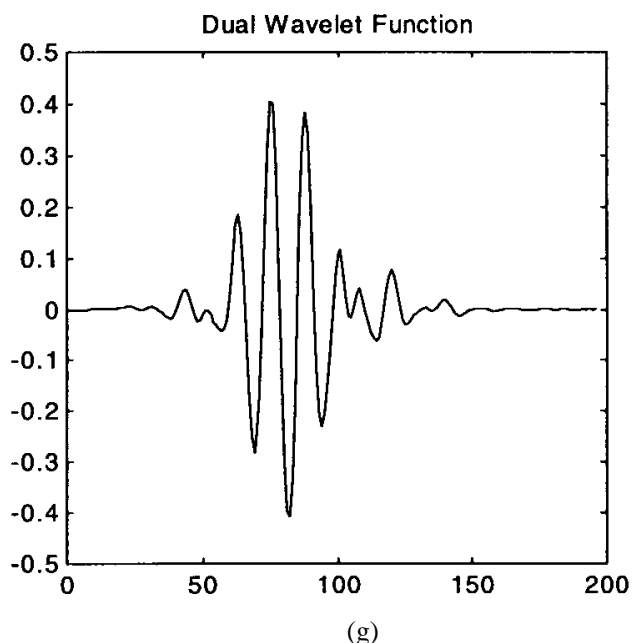

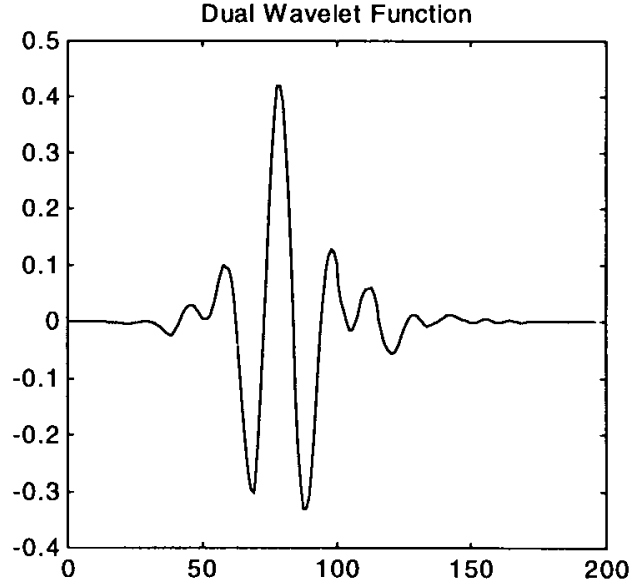

(f)

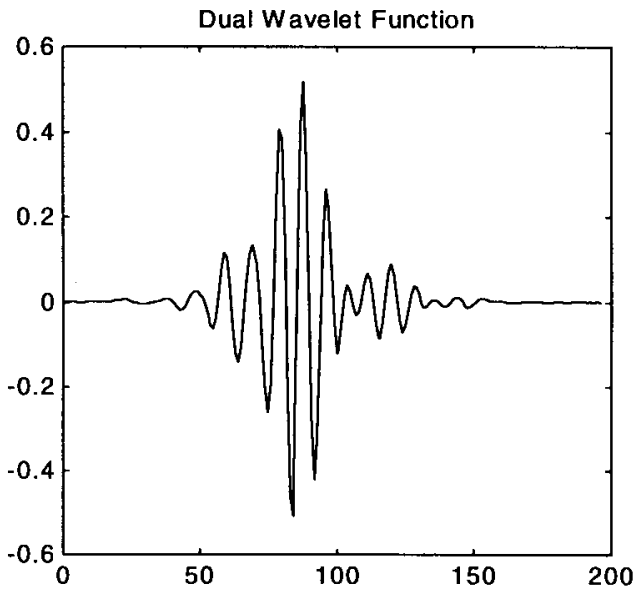

(h)

Fig. 5. (Continued.) Scaling and wavelet functions of four-channel biorthogonal CMFB wavelet bases. (e) Wavelet function-3. (f) Dual wavelet function-1. (g) Dual wavelet function-2. (h) Dual wavelet function-3.

To prove $\int \phi(x) \tilde{\phi}(x-\ell) d x=\delta_{\ell 0}$, it suffices to prove $L^{2}-$ $\lim _{n \rightarrow \infty} U_{n}(\xi)=\Phi(\xi)$ and $L^{2}-\lim _{n \rightarrow \infty} \tilde{U}_{n}(\xi)=\tilde{\Phi}(\xi)$. Now

$$
\begin{aligned}
\left|U_{n}(\xi)\right|= & \frac{1}{\sqrt{2 \pi}}\left[\frac{\sin (\xi / 2)}{M^{n} \sin \left(M^{-n} \xi / 2\right)}\right]^{L} \\
& \cdot \prod_{\ell=1}^{\infty}\left|Q\left(M^{-\ell} \xi\right)\right| \chi_{[-\pi, \pi]}\left(M^{-n} \xi\right) .
\end{aligned}
$$

On the other hand

$$
|\sin \xi| \geq \frac{2}{\pi} \mid \xi \text { for }|\xi| \leq \pi / 2 .
$$

Hence, $\left|\sin \left(M^{-n} \xi / 2\right)\right|^{-1} \chi_{[-\pi, \pi]}\left(M^{-n} \xi\right) \leq \pi M^{n}|\xi|^{-1}$, which implies

$$
\begin{gathered}
\left|\frac{\sin (\xi / 2)}{M^{n} \sin \left(M^{-n} \xi / 2\right)}\right| \chi_{[-\pi, \pi]}\left(M^{-n} \xi\right) \\
\leq 2 \pi\left|\frac{\sin (\xi / 2)}{\xi / 2}\right| \leq C(1+|\xi|)^{-1} .
\end{gathered}
$$

Writing $n=k n^{\prime}+q$ with $0 \leq q<k$,

$$
\begin{aligned}
\prod_{\ell=1}^{n} Q\left(M^{-\ell} \xi\right) & \leq\left[\sup _{\xi}|Q(\xi)|\right]^{q} \prod_{\ell^{\prime}=0}^{n^{\prime}}\left|\vartheta\left(M^{k \ell^{\prime}} \xi\right)\right| \\
& \leq\left[\sup _{\xi}|Q(\xi)|\right]^{q} C(1+|\xi|)^{\log B_{k} / \log M} .
\end{aligned}
$$

Putting it all together, we have

$$
\left|U_{n}(\xi)\right| \leq C^{\prime}(1+|\xi|)^{-L+\log B_{k} / \log M}
$$

where $C^{\prime}$ is independent of $n$. Since $U_{n}(\xi)$ converges pointwise to $\Phi(\xi)$, the Lebesgue dominated theorem implies that $U_{n}(\xi)$ tends to $\Phi(\xi)$ in $L^{2}(\Re)$. The $L^{2}$-convergence of $\tilde{U}_{n}(\xi)$ is proved analogously. Therefore, $\phi(x-\ell)$ and $\tilde{\phi}\left(x-\ell^{\prime}\right)$ are biorthogonal.

\section{APPENDIX B}

Here, we shall prove that $\Phi(\xi)$ satisfies the decay condition (4.17) if $H_{0}(\omega)$ has $K$-regularity. We will consider the case $|\xi| \leq 1$ and $|\xi| \geq 1$ separately

$$
1^{\circ}|\xi| \leq 1 \text {. }
$$

Consider

$$
\begin{aligned}
|\Phi(\xi)| & =\left|\prod_{j=1}^{\infty} H_{0}\left(M^{-j} \xi\right)\right| \leq \prod_{j=1}^{\infty}\left(1+C M^{-j}|\xi|\right) \\
& \leq \prod_{j=1}^{\infty} \exp \left(C^{\prime} M^{-j}|\xi|\right)=\exp \left(\frac{C^{\prime}|\xi|}{M-1}\right)
\end{aligned}
$$

which is uniformly bounded for $|\xi| \leq 1$. Since $\exp [C|\xi| / M-1]$ is monotonic increasing in the range $[0,1]$ and $|\Phi(\xi)| \leq C(1+$ 
$|\xi|)^{-(1 / 2)}-\varepsilon$ is monotonic decreasing in the same range, we can always choose a proper value of $C$ such that the decay condition is satisfied. Therefore, we need only to consider the case $|\xi| \geq 1$.

$2^{\circ}|\xi| \geq 1$.

Since

$$
\begin{aligned}
& \frac{1+e^{-i \xi}+e^{-2 j \xi}+\cdots+e^{-(M-1) j \xi}}{M} \\
& =\frac{1}{M} \frac{1-e^{-j M \xi}}{1-e^{-j \xi}}=\frac{e^{-j(M-1) \xi / 2}}{M} \frac{\sin (M \xi / 2)}{\sin \xi / 2}
\end{aligned}
$$

we have

$$
\begin{aligned}
& \prod_{\ell=1}^{\infty} \frac{1}{M} \frac{1-e^{-j M\left(M^{-\ell} \xi\right)}}{1-e^{-j\left(M^{-\ell} \xi\right)}} \\
& \quad=\prod_{\ell=1}^{\infty} \frac{e^{-j(M-1) M^{-\ell} \xi / 2}}{M} \cdot \frac{\sin \left[M^{-(\ell-1)} \xi / 2\right]}{\sin M^{-\ell \xi / 2}} \\
& \quad=e^{-j \xi / 2} \prod_{\ell=1}^{\infty} \frac{\sin \left[M^{-(\ell-1)} \xi / 2\right]}{M \sin M^{-\ell \xi / 2}}=e^{-j \xi / 2} \frac{\sin (\xi / 2)}{\xi / 2} .
\end{aligned}
$$

Hence, we have

$$
\begin{aligned}
\Phi(\xi) & =(2 \pi)^{-1 / 2} \prod_{j=1}^{\infty} H_{0}\left(M^{-j} \xi\right) \\
& =\exp (-j L \xi / 2)\left[\frac{\sin (\xi / 2)}{(\xi / 2)}\right]^{L} \prod_{\ell=0}^{\infty} \vartheta\left(M^{-k \ell} \xi\right)
\end{aligned}
$$

with $\vartheta(\xi)=Q(\xi / M) Q\left(\xi / M^{2}\right) \cdots Q\left(\xi / M^{k}\right)$. Since $|\xi|>1$, there exists $\ell_{0} \geq 0$ so that $M^{k \ell_{0}} \leq|\xi| \leq M^{k\left(\ell_{0}+1\right)}$. By the same argument as in the case $|\xi| \geq 1$

$$
\prod_{\ell=\ell_{0}+1}^{\infty} \vartheta\left[M^{-k \ell} \xi\right]=\prod_{i=0}^{\infty} \vartheta\left[M^{-k i} M^{-k\left(\ell_{0}+1\right)} \xi\right]
$$

is bounded independently of $\xi$ since $\left|M^{-k\left(\ell_{0}+1\right)} \xi\right| \leq 1$. On the other hand

$$
\begin{aligned}
\prod_{\ell=\ell_{0}+1}^{\infty} \vartheta\left(M^{-k \ell} \xi\right) & \leq B_{k}^{k\left(\ell_{0}+1\right)} \leq B_{k}^{k+\log |\xi| / \log M} \\
& \leq C^{\prime}(1+|\xi|)^{\log B_{k} / \log M} .
\end{aligned}
$$

Finally, we have the desired result $|\Phi(\xi)| \leq C(1+$ $|\xi|)^{-L+\log B_{k} / \log M}$.

\section{ACKNOWLEDGMENT}

The authors would like to thank Dr. K. M. Hong with the Department of Electrical and Electronic Engineering, University of Hong Kong, for useful discussions.

\section{REFERENCES}

[1] A. Grossmann and J. Morlet, "Decomposition of Hardy functions into square integrable wavelets of constant shape," Soc. Ind. Appl. Math. J. Math., vol. 15, pp. 723-736, 1984.

[2] I. Daubechies, "Orthonormal bases of compactly supported wavelets," Commun. Pure Appl. Math., vol. XLI, pp. 909-996, 1988.

[3] _ _ "The wavelet transform, time- frequency localization, and signal analysis," IEEE Trans. Inform. Theory, vol. 36, pp. 961-1005, Sept. 1990.

[4] S. G. Mallat, "Multiresolution approximations and wavelet orthonormal bases of $L^{2}(\Re)$," Trans. Amer. Math. Soc.,, vol. 315, pp. 69-88, 1989.

[5] A. Cohen, I. Daubechies, and J. Feauveau, "Biorthogonal bases of compactly supported wavelets," Commun. Pure Appl. Math., vol. 45, pp. 485-560, 1992.
[6] M. Vetterli and C. Herley, "Wavelets and filter banks: Theory and design," IEEE Trans. Signal Processing, vol. 40, pp. 2207-2232, Sept. 1992.

[7] H. Zou and A. H. Tewfik, "Discrete orthogonal $M$-band wavelet decompositions," in Proc. IEEE ICASSP, 1992, vol. IV, pp. 605-608.

[8] P. Steffen, P. N. Heller, R. A. Gopinath, and C. S. Burrus, "Theory of regular $M$-band wavelet bases," IEEE Trans. Signal Processing, vol. 41, pp. 3497-3511, Dec. 1993.

[9] P. P. Vaidyanathan, Multirate Systems and Filter Banks. Englewood Cliffs, NJ: Prentice-Hall, 1992.

[10] M. J. T. Smith and T. P. Barnwell, III, "Exact reconstruction techniques for tree-structured sub-band coders," IEEE Trans. Acoust., Speech, Signal Processing, vol. ASSP-34, pp. 434-441, June 1986.

[11] R. D. Koilpillai and P. P. Vaidyanathan, "Cosine-modulated FIR filter banks satisfying perfect reconstruction," IEEE Trans. Signal Processing, vol. 40, pp. 770-783, Apr. 1992.

[12] H. S. Malvar, "Extended lapped transforms: Properties, applications, and fast algorithms," IEEE Trans. Signal Processing, vol. 40, pp. 2703-2714, Nov. 1992

[13] T. Q. Nguyen and R. D. Koilpillai, "The theory and design of arbitrarylength cosine-modulated filter banks and wavelets, satisfying perfect reconstruction," IEEE Trans. Signal Processing, vol. 44, pp. 473-483, Mar. 1996.

[14] R. A. Gopinath, "Modulated filter banks and wavelets-A unified theory," in Proc. ICASSP, 1996, p. 1585.

[15] S. C. Chan, "Theory of $M$-channel biorthogonal compactly supported wavelets bases," Int. Rep., Univ. Hong Kong, Dec. 1996.

[16] Y. Luo, S. C. Chan, and K. L. Ho, "Theory and design of arbitrarylength biorthogonal cosine-modulated filter banks," IEEE ISCAS, 1997, p. 2429.

[17] A. H. Tewfik and M. Kim, "Fast positive definite linear system solvers," IEEE Trans. Signal Processing, vol. 42, pp. 572-585, Mar. 1994.

[18] "Special Issue on Wavelets," Proc. IEEE, vol. 84, Apr. 1996.

[19] T. Q. Nguyen and P. N. Heller, "Biorthogonal cosine-modulated filter bank," Proc. IEEE ICASSP, 1996, vol. III, pp. 1471-1474. 